\title{
The most common used antibiotic drugs among dental medicine doctors
}

\author{
Mihajlo Petrovski*, Olivera Terzieva-Petrovska \\ Faculty of medical sciences, Goce Delcev University, Krste Misirkov 10-a, 2000 Stip, Republic of N. Macedonia
}

\section{Introduction}

Antibiotics are the most commonly used drugs in everyday dental practice. By definition, antibiotics are pharmacological agents that can completely destroy pathogenic microorganisms or prevent their growth or proliferation, without causing significant damage to the host organism- human body. Antibiotics are defined as natural or synthetic organic substances that in low concentrations inhibit or kill selective micro-organisms (Davies \& Davies, 2010).

Usually the use of antibiotics in everyday dental practice is characterized by empirical uses based on clinique and bacteriologically commonly known etiological factors. Most dentists use only one antibiotic depending on their experience, but the range of prescribed antibiotics is small and is related to the period, knowledge and experience of dentists (Kaul et al., 2018). However, we are more likely witness of improper use of antibiotics, or using them into treatment where there is no need for them.

The pharmacological properties of antibiotics are crucial and critical in deciding on their use, dosage, ways and frequency of administration. Important pharmacological determinants are body weight, degree of absorption, rate of metabolism, and the duration of effective antimicrobial levels at the site of infection. The efficacy of antibiotic therapy is determined by the antimicrobial spectrum and the pharmacokinetic properties of the drug (Pallasch, 1996).

Therapeutic insufficiencies with some antibiotic medicaments are due to the presence or development of resistant types of microorganisms may be a problem that occurs in dental treatment. For example, the main indication for systemic antimicrobial therapy are those patients who do not result from conventional therapy, or patients with aggressive forms of periodontitis or associated with predisposing medical conditions Patients with acute or severe periodontal infections (periodontal abscess, acute necrotizing gingivitis or periodontitis) can also benefit from antibiotic therapy (Kapoor et al., 2012).

The main goal of this research was to assess the types and frequency of prescribed antibiotics by dentists, indications for prescribing antibiotics, as well as the knowledge of dentists regarding the use of antibiotics.

\section{Material and method}

Nine dental clinics with 14 doctors of dental medicine were included in this study. Eight from the doctors were general dentists, and the other six were dental specialist. Four from the specialist were specialists of oral surgery, one specialist of pedodontics and one for oral pathology and periodontology. Specialists in prosthodontics and orthodontics were excluded from the research.

\footnotetext{
*mihajlo.petrovski@ugd.edu.mk
} 
The total number of patients for a period of two weeks in which any type dental intervention was performed was registered. An examination was carried out regarding the data on the prescribed antibiotics over two weeks in April 2019.

Dental offices and dentist for the examination were selected randomly examination. Total numbers of ten dental offices were included in this research. During the study, (1) the number of interventions involving antibiotic, (2) the type of antibiotic used for treatment, and (3) the underlying disease due to which antibiotic was involved in the treatment were recorded.

The data obtained from the clinical examination after the collection was statistically processed. For statistical processing was used special software for statistical processing of data- Statistica 7.1.

\section{Results and discussion}

During the research period, interventions were done on 5764 patients. In 328 or $5.7 \%$ cases some type of antibiotic was prescribed. Eighty percent of Mot of the examined dentists included in the study proscribed only one type of antibiotic. In only $1.22 \%$ in the patients' population combination of antibiotics was prescribed, such as amoxicillin/clavulanic acid with metronidazole. About $20 \%$ of dentists proscribed an antibiotic without performing local treatment.

The most commonly used antibiotics in everyday dental practice in Eastern Macedonia are:

1. Doxycycline $-27.74 \%$

2. Amoxicillin + clavulonic acid $-26.52 \%$

3. Clindamycin $-22.87 \%$

4. Ampicillin - $14.02 \%$;

5. Cefalexin $-8.53 \%$.

The average number of prescribed antibiotics during one week per doctor is 3.24. None of the dentists included in the study have performed appropriate microbiological confirmation for antibiotic selection before the prescription.

According to our study the most common oral diseases where antibiotic treatment has been initiated are: periapical pathologies (most often periapical abscess) $-51.52 \%$ or 169 patients, gangrenous teeth in $3.66 \%$ or 12 patients, post-extraction wounds and infections $13.41 \%$ or 44 patients, the presence of an impacted or semi-implanted third molar teeth in
$28.66 \%$ or 94 patients and for the treatment of periodontal conditions $2.75 \%$ or 9 patients. The most common diagnosis for prescribing an antibiotic is periapical abscess at $44.81 \%$. During the evaluated period none of the dentists initiated antibiotic prescription in prophylactics.

The antibiotic selection should be primarily based on microbiological analyzes from the target zone of the inflammation, but obtained results form everyday dental practice confirmed that the selection was made empirically based on clinical signs.

\section{Conclusion}

Based on the presented data, it can be noticed that amoxicilinum + clavulic acid and doxycycline are most commonly proscribed antibiotics among dentists. Periapical pathoses was the most common cause antibiotics use in patients in our study. However, the high percentage of prescribed antibiotics indicates the tendency for irrational drug utilization. Our results can contribute in evaluation of rational antibiotics proscription among dentists as well as in developing guideline for rational dental pharmacology practice.

\section{References}

Davies, J., Davies, D., 2010. Origins and evolution of antibiotic resistance. Microbiology and molecular biology reviews 74(3), 417-433.

Kapoor, A., Malhotra, R., Grover, V., Grover, D., 2012. Systemic antibiotic therapy in periodontics. Dental Research Journal 9(5), 505-515.

Kaul, R., Angrish, P., Jain, P., Saha, S., Sengupta, A., Mukherjee, S., 2018. A survey on the use of antibiotics among the dentists of Kolkata, West Bengal, India. International Journal of Clinical Pediatric Dentistry 11(2), 122-127.

Pallasch, T.J., 1996. Pharmacokinetic principles of antimicrobial therapy. Periodontology 2000(10), 511.

Maced. Pharm. Bull. 66 (Suppl 1) 65 - 66 (2020) 